\title{
A Review of the Ergonomic Issues in the Laparoscopic Operating Room
}

\author{
Sang D. Choi, Ph.D., M.S., CSP, CPE \\ Department of Occupational \& Environmental Safety \& Health \\ Center for Occupational Safety and Ergonomics Research \\ University of Wisconsin - Whitewater \\ 800 West Main Street, \\ Whitewater WI 53190-1790, USA \\ Phone: +1 262-472-1641 Fax: +1 262-472-1091
}

Email:chois@uww.edu

Submitted December 2011. Accepted for publication August 2012.

\begin{abstract}
This review paper discusses the ergonomic challenges associated with laparoscopy in the operating room (OR) and summarizes the practical ergonomic solutions. The literature search was conducted in the fields of laparoscopy and applied ergonomics. Findings indicated that laparoscopic OR staff (surgeons, perioperative nurses and technicians) commonly experienced physical and mental ergonomic risks while working in prolonged static and awkward body positions. This study highlighted the need for more ergonomic interventions in OR environment in order to improve the efficiency of laparoscopy. Ergonomic solutions included utilizing adjustable equipment, placing computer peripherals in optimal locations, providing ergonomic instruments, and improving communication. Understanding the job- or task-related ergonomic risks and hazards could help identify intervention requirements to meet the challenges associated with increased dependency on advanced high technology in the OR.
\end{abstract}

Keywords: laparoscopy, operating room, surgeon, perioperative nurses and technicians, ergonomics

\section{INTRODUCTION}

Healthcare is one of the largest industries in the United States, and provides over 14 million jobs for wage and salary workers [1]. Employment in healthcare is expected to continue to grow, more than any other industries, largely in response to rapid expansion of the elderly population. Concurrently, the incidence of occupational injury and illness in healthcare has been higher than the average for the total private sector [1]. Ergonomic concerns in the healthcare industry continue to rise as new emerging technologies and 
procedures come about [2]. Understanding the characteristics of ergonomics-related problems are essential measures towards finding effective interventions [3].

Ergonomics is defined as applying knowledge from human sciences to match jobs, systems, products, and environments to physical and mental capabilities of people in order to promote safety, health, and wellbeing while performing tasks effectively [4]. As new technology and products arise, ergonomics has become an increasingly important aspect in the healthcare industry. Ergonomic guidelines should be exploited to enhance man-machine interface and work efficiency to reduce risk of injury and ensure employees' health $[5,6]$.

Healthcare personnel in the operating room (OR) environment are at significant ergonomic risks due to the nature of their tasks such as standing in static and awkward postures, holding equipment and materials, working long durations, using precision skills, and operating new equipment $[3,7,8]$. The instruments, devices, and equipment used in the OR may not be fitted to the worker, which can promote an onset of musculoskeletal pain [9]. Improving ergonomics in the OR environment can lead to less stress, strain, and fatigue for the worker, and help prevent musculoskeletal injuries to the surgical team and boost performance [5]. The OR staff is exposed to ergonomic risk factors that may develop work-related musculoskeletal disorders (MSDs) [3].

There is a growing body of literature on the ergonomics of minimally invasive surgery (MIS). MIS, or termed endoscopy or keyhole surgery, is performed through small incisions in the skin or through the natural opening of the human body [5, 10, 11]. The goal of most studies on ergonomics in MIS is to improve working conditions associated with the use of endoscopic hand-held instruments and video monitors [8, 12-15] while focusing on the ergonomic concerns of surgeons and laparoscopy [16-21]. However, an optimal work environment also depends on interactions between the environment, equipment, and medical staff. The increasing dependency on technology to perform surgical procedures brings forth physical, sensorial and cognitive ergonomic problems to the OR surgical team (including, e.g., surgeon, assisting surgeon, scrub nurse, circulating nurse) [5, 22, 23]. For example, the minimally invasive OR is generally crowded with essential apparatuses, thus diminishing the working space for the surgical team [10, 24]. It is desperately needed to improve the laparoscopic working environment, especially in the face of an impending shortage of OR staff (e.g., surgeons) in the US [25]. However, very few studies have provided a systematic approach to review the ergonomic impact of laparoscopy on the practicing surgeon and perioperative nurses and technicians (PNTs).

The purpose of this paper is to review the occupational ergonomic issues in the laparoscopic OR work environment, and synthesize the practical job- or task-specific ergonomic solutions associated with the laparoscopic OR.

\section{METHODS AND PROCEDURES}

An initial search was conducted to find all peer-reviewed research related to the ergonomics in the laparoscopic OR. The search was conducted primarily using electronic databases, supplemented by books and other printed materials retrieved from 
a network of libraries. Studies published in English were drawn from peer-reviewed journals, conference proceedings, edited books, and a variety of web-based sources. Electronic resources searched included: EBSCOhost, WilsonWeb, ScienceDirect, Web of Science, Academic Search Complete, Alt HealthWatch, CINAHL Plus, Health Source-Consumer Edition, Health Source-Nursing/Academic Edition, MEDLINE, SPORTDiscus, PubMed Central, and Consumer Health Complete. Keywords utilized for the electronic search included: laparoscopy, minimally invasive surgery, surgeon, surgery, endoscopy, medical personnel, perioperative, nurse, technician, operating room, ergonomics, musculoskeletal, injury, disorder, disease, symptoms, pain, discomfort, hospital, healthcare, health, upper, lower, body, back, work, work-related, or a combination of the terms. Environmental aspects are not included in the scope of this study. Two librarians were engaged in the process of identifying appropriate databases.

Most searches were conducted for the period from 2000 to 2012, with older references added later in the process. Over 110 publications were initially identified, and 82 of them selected for inclusion in this study (76 original articles, 4 books, 2 internet web links). The decision to include refereed articles for review was based on the article's title, abstract, and contents. References chosen for inclusion were those with the abstract and full article available and specifically related to ergonomics, laparoscopic OR, surgeons and PNTs. Sixty-seven and fifteen references were found for surgeons and for PNTs, respectively.

\section{LAPAROSCOPIC OPERATING ROOM}

Laparoscopy (MIS in the abdomen) is a surgical technique that is performed in the patients' abdominal cavity through small incisions and involves viewing the surgery on a monitor. MIS has developed rapidly in recent decades with increasing diversity of laparoscopic techniques and growing numbers of laparoscopically treated patients [26, 27]. Generally, MIS is accepted among surgeons, patients, and insurance companies due to its advantages [28]. Laparoscopy results in shorter hospital stay, earlier return to the workforce, less pain, better cosmetics, and better postoperative immune function $[29,30]$.

However, the laparoscopic OR staff encounter physical stress and mental strain beyond those in open surgery [3, 26, 32-34]. MIS or laparoscopic surgery involves more and larger equipment taking more floor space and promoting limited body movement and static body postures [5, 34]. Further prominent limitations are due to the technical and mechanical nature of the equipment, including loss of haptic feedback (tactile \& force), natural hand-eye coordination and dexterity [35, 36].

Many people are involved in the OR during laparoscopy, including the patient, surgeon, assistant/resident/intern, PNTs, anesthetist and nurse anesthetist, and possibly other staff members depending on the procedures. The surgeon may specialize in a specific area, such as gynecology, orthopedics, cardiology, etc. The surgeon(s) is located at the operating table and uses various instruments to operate on the patient during the laparoscopy. PNTs include the scrub nurses and circulating nurses. This review study focuses on surgeons and PNTs involved in laparoscopy. 


\subsection{Surgeons' Physical Ergonomic Challenges}

Although MIS brings many advantages to patients, laparoscopy involves a series of physical ergonomic risks for the surgeon. Laparoscopic surgeons operate with reduced access to the patient, reduced degrees of freedom in their laparoscopic instruments, prolonged static \& awkward body positions, and repetitive movements [8, 16, 37-44].

The following describes the surgeon's job- or task-related ergonomic risks associated with surgeon's physical, perceptual and cognitive challenges.

\subsubsection{Operating Table}

The height of the operating table influences the excursions of the upper extremities of the surgeon and the assistant during laparoscopy [45]. Working at the operating table poses hazards to the surgeons depending on the procedure and the surgeon's physical condition. For example, most operating tables are designed for open surgery, and may be too high for laparoscopy [46]. This inadequate table height can cause the surgeon to work in awkward body positions (abducted arms, raised/flexed shoulders, and/or ulnar deviated wrists), and physical discomfort in the surgeon's upper arms, neck and shoulders [5, 7, 47].

In laparoscopy, surgeons are exposed to risk for musculoskeletal pain in the neck, back, shoulders, arms, wrists/hands [9]. An operating table too wide promotes abduction of the trunk to reach the patient, and could cause pain in the neck, back, and lower extremities. The operating table with clamps on the side to affix accessories can obstruct the worker from standing in a desired position [9]. Visual inspection and photograph of video-assisted thoracoscopic surgery (another type of MIS) can reveal physical ergonomic problems in the upper body and the shoulders [48]. While patient is positioned in lateral decubitus most of the time, the operating table is typically twice as wide as the patient's body. Consequently, the surgeon and the assistant have to lean over in order to operate. Additionally, excessive operating table height causes extreme abduction of the arms and ulnar deviation of the wrist. Due to the foot of the operating table, the foot pedals and cables on the floor, there is little space for the feet of surgical staff, causing a static body position. This problem is worsened by the precision actions required for thoracoscopic surgery and the continuous need to watch the monitor [48].

\subsubsection{Position of the Monitor}

The position of the viewing monitor influences the postures of the surgeon and the assisting surgeon $[45,49]$. Inadequate monitor position and height can cause physical body discomfort [50]. In laparoscopy, if the monitor is not correctly positioned in line with the operating field, a long duration of static neck flexion, extension and rotation can occur [7]. The surgeon also needs to abduct the upper body (side bending or forward bending) in order to look at the monitor if other OR staff obstruct the view. In laparoscopy performed in a traditional OR, the operative field is visualized indirectly with a laparoscope connected to a camera that projects a two-dimensional image on a monitor. The monitor is positioned at a certain height and distance outside the sterilized operating field, forcing the surgeon to work in one direction while viewing in another. If the monitor is fixed on top of a trolley (although in state-of-the-art ORs, the monitors 
are installed on the ceiling mounted booms), the adjustment possibilities of the monitor may be very limited in terms of both height and direction $[48,49,51]$.

\subsubsection{Hand-Held Instruments}

The hand-held instruments were examined using questionnaire/interview and checklist to evaluate OR ergonomic deficiencies [9]. It was noted that the laparoscopic surgeons are exposed to the ergonomic risks (e.g., excessive excursions and awkward postures) and pressure peaks on both fingers and palm $[9,45]$ that can contribute to musculoskeletal injuries [52]. These instruments include (but are not limited to) endoscopes, graspers, scissors, staplers, retractors, and irrigation tools. Surgeons experience discomfort in the arms and hands due to using surgical instruments, and forceful exertions and repetitive upper limb movements were linked to wrist and hand pain [3, 53]. A common musculoskeletal disorder called laparoscopist's thumb (surgical thumb) occurs in surgeons when the nerves of the thumb are damaged in performing laparoscopy [54].

Due to the length of the instruments and the fixed insertion point, the surgeon often has to adopt large ranges of motion in order to direct the instrument to the desired location inside the patient's body. In such non-ergonomic motions the upper limb position is awkward and can lead to increased muscle fatigue and degradation of performance [8, 13, 55-57]. Changing instruments and repositioning within the abdomen are cumbersome and time-consuming tasks, because of the need to introduce the instruments through the trocar $[13,58]$. Compared with instruments used in open surgery, the laparoscopic instruments require substantially greater muscle forces and more awkward wrist movements [48, 49]. Laparoscopic instruments are characterized by an inauspicious force transmission from the handle to the tip so that the laparoscopic surgeons need to apply 4-6 times more force to complete the same task as in conventional surgery [49].

\subsubsection{Handle}

The surgeon-instrument interface is largely influenced by the instrument handle design which determines the configuration of the surgeon's hand grip, and hence, the overall efficiency and comfort [59-61]. Often, the instruments' handles is designed with a fixed size for all surgeons and for all tasks. The contact surfaces, that are usually relatively narrow, the handles' shapes, and the movements do not correspond with anatomic features of the surgeon's hand. The nature of the handle and its associated activation mechanism affect joint movements, muscle recruitment, and muscle fatigue in the upper arm. In turn, these factors impact on the surgeon's comfort level, the execution speed, and the quality of task performance. Excessive pressure on sensitive areas of the palm and fingers can cause temporary nerve injuries $[8,15,17,55]$. Moreover, the majority of current laparoscopic instruments have pistol grips with finger rings, which induce extreme ulnar deviation and wrist flexion. Pistol-grip finger loop handles force the arms into abduction to enable the thumb to enter the instrument ring along the transverse plane $[12,17]$. Also, the thumb ring is usually located on the mobile limb of the instrument. The distal ring of the instrument is fixed and serves to stabilize the instrument with the other fingers. As the instrument is activated, force is transmitted by 
the thumb to the handle ring and ultimately to the tip of the instrument. This action causes the lateral aspect of the thumb to lie forcibly against the ring of the handle, resulting in trophic skin changes and in some cases nerve compression [62].

\subsubsection{Hand Force}

Current laparoscopic instrument design involved activation of the jaws by the proximal (thumb) instrument ring. The force to open and close the instrument is applied by thumb apposition alone (countered by the other fingers), in contrast to open-surgery instrument wherein the thumb and the fingers exert the force simultaneously in opposing directions [63]. As a result of these constraints, the surgeon is forced to assume atypical nonphysiologic postures for extended periods of time. Instrument length influences the overall handling of the instrument, especially when compensatory mechanisms other than increase muscle activation can be used [64]. Reported studies on muscle activation have established specific patterns for standard laparoscopic tasks [55, 64]. The most important finding of these studies is that use of current long straight laparoscopic instruments involves the recruitment of shoulder and upper arm muscle groups much more than forearm muscles. In general, longer instruments result in greater muscular recruitment and work to predispose to both fatigue and chronic injury [57]. Laparoscopic tasks resulted in significantly higher electromyographic (EMG) amplitudes in the upper-extremity muscles (thumb, forearm flexor, and deltoid) compared with the use of open instruments [39].

\subsubsection{Foot Pedals}

In laparoscopy, diathermy and ultrasonic equipment is operated by means of one or more foot pedals positioned on the floor in front of the surgeon [45]. Surgeons use foot pedals to control the diathermy - the procedure where electrically heated probes cauterize blood vessels and tissues to seal them. These foot pedals may cause several physical ergonomic concerns (e.g., discomfort in the legs and feet) while the surgeon keeps the operating foot dorsiflexed over the pedals and loads the body weight on the other foot to prevent losing contact with the pedals [50, 65]. In addition, foot pedals restrict the surgeon from moving around and promote static body postures [45].

\subsection{Surgeons' Sensorial Ergonomic Challenges}

Laparoscopy is associated with perceptual and sensorial ergonomic problems in visual displays (e.g., monitors), tactile displays (e.g., force feedback in the handle), and auditory displays (e.g., beeps and alarms in the OR) [5, 18, 48]. A specific problem of touching occurs in the palpation of the organs as there is no direct manual contact with the tissue. The sensory feedback for commercially available instruments (e.g., reusable and disposable laparoscopic dissectors) was low compared to bare fingers [66]. Another sensorial ergonomic issue may occur in the use of the foot pedals to manage the high frequency electrosurgery and the ultrasonic dissection. These pedals are situated under operating table and covered by sterile sheets. The surgeon has to look down to situate them. Both the foot pedal for electrosurgery and that for ultrasonic dissection have two switches for different functions. These two switches cannot be distinguished by touch, 
and can possibly result in dangerous situations such as hitting the wrong switch [67]. Also, the pedals can be unintentionally moved (kicked) around under the operating table, that the surgeon loses contact with the pedal and has to look down to restore the right position of the foot [68].

\subsection{Surgeons' Cognitive (Mental) Ergonomic Challenges}

Laparoscopic surgeons encounter unique cognitive ergonomic challenges due to reduced access to the patient, fewer degrees of freedom in their interventions, and lack of direct visualization of the surgical field [10, 26, 48]. In laparoscopy, the twodimensional viewing of the three-dimensional field requires continuous reflection on an interpretation of the images $[10,13,17]$. When the viewing direction of the surgeon differs from the viewing direction of the camera, the surgeon has to translate the images on the monitor during the video-assisted surgery [48]. Another quandary that limits the surgeon's acquisition of laparoscopic surgical skills and jeopardizing the surgical quality is due to the fulcrum effect as an internal movement to the right is displayed as a movement to the left on the monitor. For an inexperienced surgeon, this can result in a significantly poorer performance [23]. Comparing laparoscopic and open surgical techniques with regard to performing knot tying tasks, the laparoscopic approach resulted in fewer tied knots and correlated with significantly higher mental stress [31].

\subsection{PNTs' Physical Ergonomic Challenges}

The job- or task-related ergonomic risks (physical and mental) associated with PNTs in the laparoscopic OR work environment include those related to surgical carts, operating table, foot pedal controls, hand tool use, and viewing monitors.

\subsubsection{Surgical Tray Carts}

Carrying surgical trays can be physically exhausting. Each tray contains surgical instruments and weighs approximately $8-11 \mathrm{~kg}$; the PNTs are required to lift the trays off carts. Pushing and pulling these surgical tray carts as well as carrying the trays can contribute to upper and lower extremity musculoskeletal pain. Circulating and scrub nurses are typically standing throughout the procedure, causing lower extremity discomfort [69]. The current instrument tables and the working surfaces of the scrub nurse are all placed beside and behind the scrub nurse. (Even the mayo instrument table placed over the operating table surface is not located directly in front of the scrub nurse but at the side.) Reaching for the instruments on the instrument tables implies frequent torsion of the upper body. Further, turning the whole body to reach the instruments on the third instrument table, positioned at the backside of the scrub nurse, takes too much time and requires extreme torsion of the upper body [70].

\subsubsection{Operating Table}

The optimal operating table height for scrub nurses and surgeons differ because of different user group demographics and position at the operating table. The surgeons, forming the primary user group, usually dictate the operating table heights [21]. The operating table draws similar complaints from the PNTs as from the surgeons. The 
scrub nurse is exposed to risk for musculoskeletal pain in the neck, back, shoulders, upper arms, wrists/hands, and lower extremities due to problems with operating tables (too wide or too high/low), restricted space at the operating table, and excessive exertion moving patient between stretcher and operating table. Also, the clamps to affix accessories on the side of operating table can create pressure points and bruise the soft tissue around the pelvis if the staff leans against them $[5,9]$.

\subsubsection{Monitor Placement}

In laparoscopy, the scrub nurse is often not able to have a clear vision of the monitor. Monitor placement mostly focuses on the comfort of the surgeon [15]. During laparoscopy, the scrub nurse is exposed to risk for musculoskeletal discomfort or pain in the neck, back, and upper extremities due to the improper monitor placement and position [9]. Additionally, prolonged standing and adjusting the body position to optimize vision on the operating field initiates leaning at the thigh level against the operating table, and causes discomfort in the thighs [70].

\subsection{PNTs' Mental Ergonomic Challenges}

PNTs are exposed to high mental ergonomic risks in the OR environment [69]. Working fast rotating shifts impaired PNTs' perceptual and motor abilities [71]. Some ORs have not been updated or remodeled for decades, and continue to be used without appropriate design changes to accommodate new technology. PNTs perceived frequent medical technology changes, work role overload and ambiguity, lack of equipment, and inadequate training specifically among scrub nurses, circulating nurses, and operating theater managers contribute to the increased mental stress in the OR environment $[5,72,73]$. Violence and traffic patterns in the OR have also been investigated and reported as factors causing additional levels of stress in PNTs [74]. Moreover, PNTs perceived that lack of appropriate communication (e.g., poor communication between surgeons and PNTs, and poor communication among the staff concerning missing instruments) in the OR results in mental stress [69].

\section{DISCUSSION AND RECOMMENDATION}

MIS has developed rapidly in recent decades with increasing diversity of laparoscopic procedures and growing numbers of laparoscopically treated patients. This study reviews and consolidates the existing research literature on the ergonomic considerations of laparoscopic OR. Table 1 summarizes the occupational ergonomic challenges reviewed in this study, and describes the suggested solutions.

As shown in Table 1, there are significant ergonomic challenges associated with the laparoscopic OR. Working in long durations of static and awkward postures in a laparoscopic OR environment can increase risk of developing MSDs [42-44]. For instance, the musculoskeletal health of surgeons was threatened by the inappropriate operating table height/width, foot pedal controls, hand tool use, and viewing monitors. PNTs experienced musculoskeletal problems from not only the operating table but from pushing and pulling surgical carts and carrying surgical trays. Common MSD risks between the surgeons and PNTs are found in the neck, back, and upper body associated 


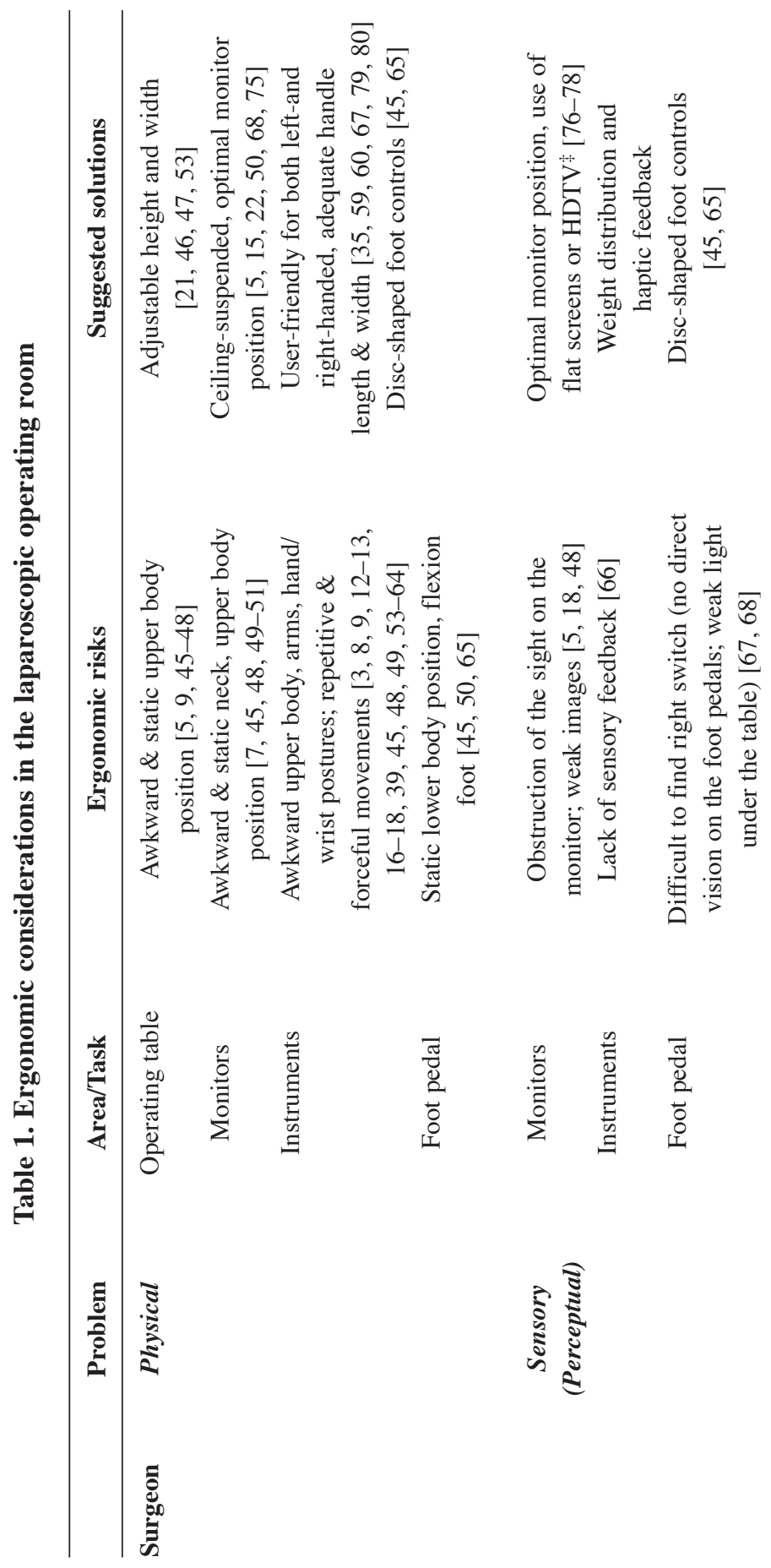

ฮัฐ 


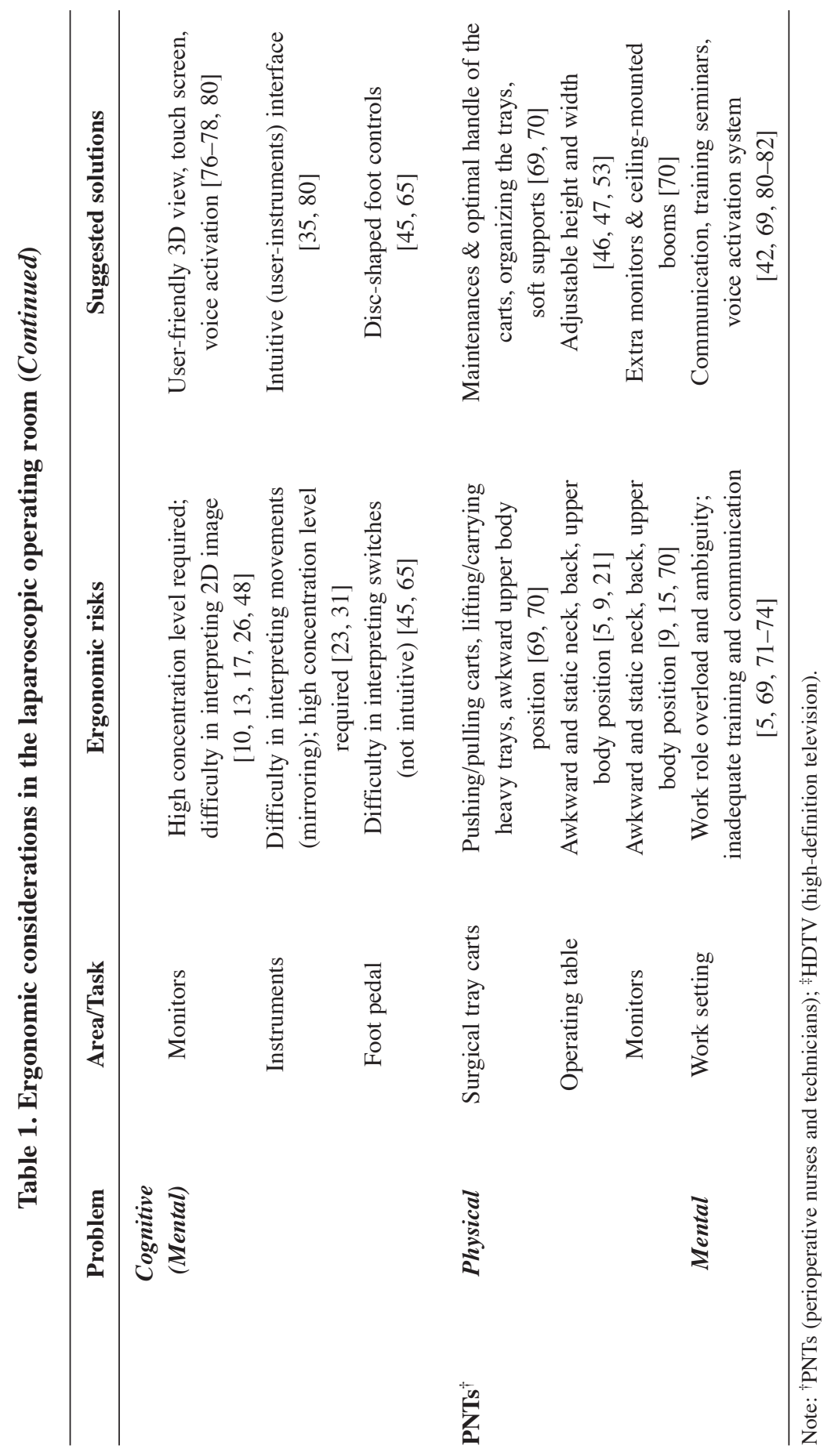


with the static and awkward body positions (see Table 1). Recommendations to mitigate the ergonomic risks associated with the laparoscopic OR environment are discussed below.

\subsection{Adjustable Operating Table}

Implementing an adjustable operating table can help reduce awkward postures while performing laparoscopy. Lowering the height of the operating table to counterbalance the greater length of the laparoscopic instruments in advance of the operation is a practical solution. New operating tables with a much greater adjustability, ranging from $29 \mathrm{~cm}$ to $122 \mathrm{~cm}$ above floor level, are needed to accomplish optimal working height for different designs of instrument handles [46, 47]. An optimal operating table height will also mitigate problems associated with manipulating instruments. The discomfort and difficulty were lowest when handles were positioned at elbow height [47]. The operating surface height influences the (extreme) upper joint excursions of the surgeon. The ergonomically optimal operating surface height reduces the discomfort in the shoulders, back, and wrists of the surgeon during laparoscopy [21]. Additionally, the sides of the operating table should be padded with a foam hip support to prevent bruising tissue when leaned against [53].

\subsection{Viewing Monitor}

The viewing monitor should be properly positioned to be seen in a neutral posture; this can help prevent fatigue and musculoskeletal injuries [50]. The members of the surgical team stand on both sides of the operating table during the majority of the procedures. From an ergonomic point of view, each member of the surgical team should have an unobstructed line of vision without neck torsion [22]. The optimum monitor height should be below eye level, resulting in a slight flexion of the head (between 15-45 from the horizontal) without torsion of the neck and back $[68,75]$. If multiple monitors are used during the procedure, they should be strategically placed to minimize blocking the view of all OR staff. Also, the scrub nurse is often not able to have a clear vision of the monitor. Monitor placement is mostly based on consideration of the comfort of the surgeon $[5,15]$. A solution may be additional monitors and installation of monitors on ceiling-mounted booms to allow optimal vision of the scrub nurse [70]. Flat screen and high-definition television (HDTV) with higher brightness and more detailed picture can improve the visual quality in the OR [76-78].

\subsection{Hand-Held Instruments}

To reduce discomfort and musculoskeletal pain in the upper extremities, surgeons should avoid using instruments for tasks other than what they are originally intended for [60]. User-friendly instruments for both left- and right-handed surgeons can help decrease awkward postures and the urge to wrongfully use them. The handle should have an adequate gripping width in order to reduce excessive pressure. For example, using new handles for a laparoscopic dissector, the surgeon's wrist excursions remain in the ergonomically allowed zone, and that the shape of the new handle is comfortable both during precision and force manipulation [79]. When the handle is manipulated 
with a force or precision grip, wrist excursion must be neutral for $70 \%$ of the manipulation time. The angle between the handle and shaft should be between $14^{\circ}$ and $50^{\circ}$ to facilitate comfortable posture of the hand and arm [67]. Additionally, ergonomic weight distribution, intuitive interface and haptic feedback of the hand-held instrument should be considered to optimize efficiency of the surgery $[35,59,80]$.

\subsection{Disc-Shaped Foot Controls}

To prevent constant extreme dorsiflexion of the foot (i.e., more than $25^{\circ}$ ) when controlling the diathermy foot pedal, a flat round disc pedal can be employed [45, 65]. The switch is activated by positioning the foot on the disc and rotating the foot (leg): clockwise rotation activates the coagulation function, and counterclockwise rotation activates the cutting function. Since the disc is flat and thin, the user can stand on the disc during surgery with the weight spread evenly over both feet. Physical discomfort can be reduced because no flexion of the foot is needed and the rotations needed for control are not frequent [45]. Moreover, the pedal does not obstruct the freedom of movements, and activation of the wrong switch can be avoided [65].

\subsection{Surgical Tray Carts}

Surgical carts need to be properly maintained with a proper handle installed to reduce the force required for pushing and pulling. This can help reduce the injuries to the lower extremities in PNTs and can significantly reduce loss of time [69]. Better methods of identifying the trays for each surgical procedure and the proper organization of the trays would reduce the frequency of lifting the trays. PNTs need to identify specific trays and transfer them to smaller carts to be used in a specific surgical procedure [69]. The scrub nurse should rearrange the surgical carts in order to avoid extreme awkward posture (reaching for instruments on the instrument tables located at the backside). Soft supports on resting points of instrument tables or on the operating table can help the scrub nurse to remain in the neutral standing position and to decrease pressure and chance of bruises [70].

\subsection{Communication and Training}

Laparoscopic OR design to provide surgeons with direct access and control of surgical devices would be beneficial to both surgeons and nurses. Surgeons can access to the critical devices via methods such as touch screen control and voice activation [80]. Voice activation gives surgeons immediate access to and direct control of surgical devices, and provides the OR team with critical information. Many devices can now be controlled by voice activation software, including cameras, light sources, digital image capture and documentation devices, printers, insufflators, OR ambient and surgical lighting systems, operating tables, and electrocauteries [80]. Good communication is needed to reduce unnecessary work $[69,81]$. (When PNTs are not aware of the surgeon's needs, confusion and disorganization occur.) Training program or seminars to improve the OR staff's familiarity with and performance of new MIS techniques can be beneficial to both surgeons and PNTs. Furthermore, a team of PNTs specially designated for MIS procedures can accomplish tasks more quickly and efficiently than a random group of circulating nurses and scrub technicians [42, 80, 82]. 


\section{CONCLUSION}

The increasing dependency on technology in the laparoscopy has created physical and mental ergonomic challenges. The laparoscopic OR staff (surgeons and PNTs) commonly experience prolonged static body postures, extreme joint positions and repetitive movements. The most affected body parts are the neck, back, shoulders, and wrists/hands due to the frequent postures held while performing fine hand movement for surgery or technical work. The common sources in the OR contributing to the ergonomic problems include operating table, foot control pedals, footstools, various hand tools, surgical monitors, surgical trays and carts.

With the ever growing variety of technology, laparoscopy ergonomics research is lagging behind the pace of new OR surgical procedure development. Ergonomic researchers' input should be incorporated in the development of surgical instruments and their manufactures. More attention is warranted to promote better ergonomics in laparoscopy by encouraging the industry to provide multidisciplinary human-centered design approach for the improvement of ergonomics, comfort, and usability for the broad range of OR medical team.

\section{REFERENCES}

[1] Bureau of Labor Statistics. U.S. Department of Labor, Occupational Outlook Handbook, 2012-13 Edition, Career Guide to Industries. 2010. http://www.bls.gov/ooh/about/career-guide-toindustries.htm. Accessed July 30, 2012.

[2] Verdaasdonk EG, Stassen LP, van der Elst M, Karsten TM, Dankelman J. Problems with technical equipment during laparoscopic surgery: an observational study. Surgical Endoscopy. 2007, 21:275-279.

[3] Szeto G, Ho P, Ting A, Poon J, Cheng S, Tsang R. Work-related musculoskeletal symptoms in surgeons. Journal of Occupational Rehabilitation. 2009, 19:175-184.

[4] Wilson J R, Corlett N. Evaluation of Human Work, $3^{\text {rd }}$ ed., Taylor \& Francis, Boca Raton, FL, 2005.

[5] Wauben LS, Albayrak A, Goossens RHM. Ergonomics in the Operating Room - An Overview, In: Brinkerhoff BN (ed.), Ergonomics: Design, Integration, and Implementation, Nova Science Publishers, NY, 2009, 79-118.

[6] National Institute for Occupational Safety and Health. Ergonomic Guidelines for Manual Material Handling, DHHS Publication No. 2007-131, Washington, DC: US, Department of Health and Human Services, 2007.

[7] Berguer R, Rab G, Abu-Ghaida H, Alarcon A, Chung J. A comparison of surgeons' posture during laparoscopic and open surgical procedures. Surgical Endoscopy. 1997, 11:139-142.

[8] Berguer R. Surgical technology and the ergonomics of laparoscopic instruments. Surgical Endoscopy. 1998, 12:458-462.

[9] Koneczny S. The operating room: architectural conditions and potential hazards. WORK. 2009, 33:145-164.

[10] Van Veelen MA, Nederlof EAL, Goossens RHM, Schot CJ, Jakimowicz JJ. Ergonomic problems encountered by the medical team related to products used for minimally invasive surgery. Surgical Endoscopy. 2003, 17:1077-1081.

[11] Patkin M. History of ergonomy in surgery. In Bruch HP, Kockerling F, Bouchard R, Schuss-Pass C (Eds), New Aspects of High Technology in Medicine, Monduzzi Editore, Hanover, Germany, 2000:239-46.

[12] Berguer R, Gerber S, Klipatrick G, Remler M, Beckley DA. Comparison of forearm and thumb muscle electromyographic responses to the use of laparoscopic instruments with either a finger grasp or a palm grasp. Ergonomics. 1999, 42:1634-1645. 
[13] Matern U, Waller P. Instruments for minimally invasive surgery: principles of ergonomic handles. Surgical Endoscopy. 1999, 13:174-182.

[14] Hanna GB, Shimi SM, Cuschieri A. Task performance in endoscopic surgery is influenced by location of the image display. Annals of Surgery. 1998, 227:481-484.

[15] Van Veelen MA, Jakimowicz JJ, Goossens RHM, Meijer DW, Bussmann JB. Evaluation of the usability of two types of image display systems during laparoscopy. Surgical Endoscopy. 2002, 16:674-678.

[16] Berguer R, Gerber S, Kilpatrick G, Beckley D. An ergonomic comparison of in-line vs pistol-grip handle configuration in a laparoscopic grasper. Surgical Endoscopy. 1998, 12:805-808.

[17] Berguer R, Forkey DL, Smith WD. Ergonomic problems associated with laparoscopic surgery. Surgical Endoscopy. 1999, 13(5):466-468.

[18] Goossens RHM, Van Veelen MA. Assessment of ergonomics in laparoscopic surgery. Minimally Invasive Therapy \& Allied Technology. 2001, 10:175-179.

[19] Schurr MO, Buess GF, Wieth F, Saile HJ, Botsch M. Ergonomic surgeon's chair for use during minimally invasive surgery. Surgical Laparoscopy, Endoscopy \& Percutaneous Techniques. 1999, 9:244-247.

[20] Van Veelen MA, Meijer DW, Goossens RHM, Snijders CJ. New ergonomic design criteria for handles of laparoscopic dissection forceps. Journal of Laparoendoscopic \& Advanced Surgical Techniques. 2001, 11(1):17-26.

[21] Van Veelen MA, Kazemier G, Koopman J, Goossens RHM, Meijer DW. Assessment of the ergonomically optimal operating surface height for laparoscopic surgery. Journal of Laparoendoscopic \& Advanced Surgical Techniques. 2002, 12(1):47-52.

[22] Albayrak A, Kazemier G, Meijer DW, Bonjer HJ. Current state of ergonomics of Dutch hospitals in the endoscopic era. Minimally Invasive Therapy and Allied Technology. 2004, 13(3):156-160.

[23] Gallagher AG, Smith CD. Human-factors lessons learned from the minimally invasive surgery revolution. Seminars in Laparoscopic Surgery. 2003, 10(3):127-139.

[24] Berguer R. The application of ergonomics in the work environment of general surgeons. Reviews on Environmental Health. 1997, 12:99-106.

[25] Polk HC, Vitale DS, Qadan M. The very busy urban surgeon: another face of the evermore obvious shortage of general surgeons. The American College of Surgeons. 2009, 209:144-147.

[26] Park A, Lee G, Seagull FJ, Meenaghan N, Dexter, D. Patients benefit while surgeons suffer: An impending epidemic. The American College of Surgeons. 2010, 210(3):306-313.

[27] Stomberg MW, Tronstad SE, Hedberg K, Bengtsson J, Jonsson P, Johansen L, Lindvall B. Workrelated musculoskeletal disorders when performing laparoscopic surgery. Surgical Laparoscopy, Endoscopy \& Percutaneous Techniques. 2010, 20(1):49-53.

[28] Loring B, Lemieux EA. User research improves laparoscopic instruments. Ergonomics in Design. 2010, 18:18-23.

[29] Fuchs KH. Minimally invasive surgery. Endoscopy. 2002, 34:154-159.

[30] Nilsson G, Larsson S, Johnsson F. Randomized clinical trial of laparoscopic versus open fundoplication: blind evaluation of recovery and discharge period. British Journal of Surgery. 2000, 87:873-878.

[31] Berguer R, Smith WD, Chung YH. Performing laparoscopic surgery is significantly more stressful for the surgeon than open surgery. Surgical Endoscopy. 2001, 15:1204-1207.

[32] Schwaitzberg SD, Godinez C, Kavic SM, Sutton E, Worthington RB, Colburn B, Park A. Training and working in high-stakes environments: lessons learned and problems shared by aviators and surgeons. Surg Innov. 2009, 16(2):187-195.

[33] Zheng B, Rieder E, Cassera MA, Martinec DV, Lee G, Panton ON, Park A, Swanström LL. Quantifying mental workloads of surgeons performing natural orifice transluminal endoscopic surgery (NOTES) procedures. Surgical Endoscopy, 2012, 26(5):1352-1358. 
[34] Alarcon A, Berguer RA. Comparison of operating room crowding between open and laparoscopic operations. Surgical Endoscopy. 1996, 10:916-919.

[35] Coles T, Meglan D, John N, The role of haptics in medical training simulators: A survey of the state of the art, IEEE Transactions on Haptics, 2011, 4(1):51-66.

[36] Satava RM, Bowersox JC, Mack M, Krummel TM. Robotic surgery: state of the art and future trends. Contemporary Surgery. 2001, 57:489-499.

[37] Matern U. Ergonomic deficiencies in the operating room: examples from minimally invasive surgery. WORK: A Journal of Prevention, Assessment, and Rehabilitation. 2009, 33:165-168.

[38] Matern U, Kuttler G, Giebmeyer C, Waller P, Faist M. Ergonomic aspects of five different types of laparoscopic instrument handles under dynamic conditions with respect to specific laparoscopic tasks: an electromyographic-based study. Surgical Endoscopy. 2004, 18(8):1231-1241.

[39] Berguer R, Chen J, Smith WD. A comparison of the physical effort required for laparoscopic and open surgical techniques. Archives of Surgery. 2003, 138:967-970.

[40] Emam TA, Hanna G, Cuschieri A. Ergonomic principles of task alignment, visual display, and direction of execution of laparoscopic bowel suturing. Surgical Endoscopy. 2002, 16:267-271.

[41] Pérez-Duarte FJ, Sánchez-Margallo FM, Díaz-Güemes I, Sánchez-Hurtado MA, Lucas-Hernández M, Usón Gargallo J. Ergonomics in laparoscopic surgery and its importance in surgical training. Cir Esp. 2011, June 22.

[42] Reddy, P.P., Reddy TP, Roig-Francoli J, Cone L, Sivan B, DeFoor WR, Gaitonde K, Noh PH. The Impact of the Alexander technique on improving posture and surgical ergonomics during minimally invasive surgery: pilot study. The Journal of Urology. 2011, 186(4):1658-1662.

[43] Szeto G, Cheng S, Poon J, Ting A, Tsang R, Ho P. Surgeons' static posture and movement repetitions in open and laparoscopic surgery. Journal of Surgical Research. 2012, 172(1):e19-e31.

[44] Youssef Y, Lee G, Godinez C, Sutton E, Klein RV, George IM, Seagull FJ, Park A. Laparoscopic cholecystectomy poses physical injury risk to surgeons: analysis of hand technique and standing position. Surg Endosc. 2011, 25(7):2168-2174.

[45] Van Veelen MA, Jakimowicz JJ, Kazemier G. Improved physical ergonomics of laparoscopic surgery. Minimally Invasive Therapy \& Allied Technology. 2004, 13(3):161-166.

[46] Matern U, Waller P, Giebmeyer C, Ruckauer K, Farthmann E. Ergonomics: requirements for adjusting the height of the laparoscopic operating tables. Journal of the Society of Laparoendoscopic Surgeons. 2001, 5:7-12.

[47] Berguer R, Smith WD, Davis S. An ergonomic study of the optimum operating table height for laparoscopic surgery. Surgical Endoscopy. 2002, 16:416-421.

[48] Kranenburg L, Gossot D. Ergonomic problems encountered during video-assisted thoracic surgery. Minimally Invasive Therapy \& Allied Technology. 2004, 13(3): 147-155.

[49] Nguyen NT, Ho HS, Smith WD, Philipps C, Lewis C, De Vera RM, Berguer R. An ergonomic evaluation of surgeons' axial skeletal and upper extremity movements during laparoscopic and open surgery. The American Journal of Surgery. 2001, 182(6):720-724.

[50] Wauben LS, Van Veelen MA, Gossot D, Goossens RHM. Application of ergonomic guidelines during minimally invasive surgery: a questionnaire amongst 284 surgeons. Surgical Endoscopy. 2006, 20(8):1268-1274.

[51] Zehetner J, Kaltenbacher A, Wayand W, Shamiyeh A. Screen height as an ergonomic factor in laparoscopic surgery. Surgical Endoscopy. 2006, 20:139-141.

[52] National Institute for Occupational Safety and Health. 1997. Musculoskeletal Disorders and Workplace Factors, U.S. Department of Health and Human Services. http://www.cdc.gov/ niosh/docs/97-141/. Accessed August 31, 2012.

[53] Matern U, Koneczny S. Safety, hazards and ergonomics in the operating Room. Surgical Endoscopy. 2007, 21:1965-1969.

[54] Lotz JC. The suffering surgeon. Surgical Endoscopy, 1997, 11:548. 
[55] Emam TA, Frank TG, Hanna GB, Cuschieri A. Influence of handle design on the surgeon's upper limb movements, muscle recruitment, and fatigue during endoscopic suturing. Surgical Endoscopy. 2001, 15:667-672.

[56] Patil PV, Hanna GB, Frank TG, Cuschieri A. Effect of fixation of shoulder and elbow joint movement on the precision of laparoscopic instrument manipulations. Surgical Endoscopy. 2005, 19:366-368.

[57] Uhrich ML, Underwood RA, Standeven JW, Soper NJ, Engsberg JR. Assessment of fatigue, monitor placement, and surgical experience during simulated laparoscopic surgery. Surgical Endoscopy. 2002, 16:635-639.

[58] Mehta NY, Haluck RS, Frecker MI, Snyder AJ. Sequence and task analysis of instrument use in common laparoscopic procedures. Surgical Endoscopy. 2002, 16:280-285.

[59] Lin CJ, Chen HJ, Lo YC. Ergonomic investigation of weight distribution of laparoscopic instruments. Journal of Laparoendosc Advanced Surgical Technology. 2011, 21(5):411-415.

[60] Reyes DAG, Tang B, Cuschieri A. Minimal access surgery (MAS)-related surgeon morbidity syndromes. Surgical Endoscopy. 2006, 20:1-13.

[61] Berguer R, Hrelijac A. The relationship between hand size and difficulty using surgical instruments: a survey of 726 laparoscopic surgeons. Surgical Endoscopy. 2004, 18:508-512.

[62] Lee WJ, Chae YS. Superficial nerve damage of thumb of laparoscopic surgeon. Surgical Laparoscopy, Endoscopy \& Percutaneous Techniques. 2001, 11:207-208.

[63] Verma GR. Pressure sore and digital neuropraxia of the thumb in laparoscopic cholecystectomy. Surgical Laparoscopy, Endoscopy \& Percutaneous Techniques. 2004, 14:178-179.

[64] Quick NE, Gillette JC, Shapiro R, Adrales GL, Gerlach D, Park AE. The effect of using laparoscopic instruments on muscle activation patterns during minimally invasive training procedures. Surgical Endoscopy. 2003, 17:462-465.

[65] Van Veelen MA, Snijders CJ, Van Leeuwen E, Goossens RH, Kazemier G. Improvement of foot pedals used during surgery based on new ergonomic guidelines. Surgical Endoscopy. 2003, 17(7):1086-1091.

[66] den Boer KT, Herder JL, Sjoerdsma W, Meijer DW, Gouma DJ, Stassen HG. Sensitivity of laparoscopic dissectors: what can you feel? Surgical Endoscopy. 1999, 13(9):869-873.

[67] Van Veelen MA, Meijer DW, Uijttewaal I, Goossens RHM, Snijders CJ, Kazemier G. Improvement of the laparoscopic needle holder based on new ergonomic guidelines. Surgical Endoscopy. 2003, 17:699-703.

[68] Matern U, Faist M, Kehl K, Giebmeyer C, Buess G. Monitor position in laparoscopic surgery. Surgical Endoscopy. 2005, 19:436-440.

[69] Sheikhzadeh A, Gore C, Zuckerman J, Nordin M. Perioperating nurses and technicians' perceptions of ergonomic risk factors in the surgical environment. Applied Ergonomics. 2009, 40:833-839.

[70] Gerbrands A, Albayrak A, Kazemier G. Ergonomic evaluation of the work area of the scrub nurse. Minimally Invasive Therapy \& Allied Technology. 2004, 13(3): 142-146.

[71] Chang YS, Wu YH, Hsu CY, Tang SH, Yang LL, Su SF. Impairment of perceptual and motor abilities at the end of a night shift is greater in nurses working fast rotating shifts. Sleep Medicine. 2011, 12(9):866-869.

[72] Johnston PL. Occupational stress in the operating theatre suite: should employers be concerned? Australian Health Review. 1999, 22:60-80.

[73] Sauter MA, Boyle D, Wallace D, Andrews JL, Johnson MS, Bates M, Edenfield SM, Carr R, Campbell L, Hamilton BK, Taunton RL. Psychometric evaluation of the Organizational Job Satisfaction Scale. J. Nurs. Meas. 1997, 5:53-69.

[74] Girard NJ. Does violence still occur in the OR? AORN J. 2004, 80:407-408.

[75] Kroemer KHE, Kroemer HB, Kroemer-Elbert KE, Ergonomics: How to Design for Ease and Efficiency, $2^{\text {nd }}$ ed., Prentice Hall, Inc., New Jersey, 2001.

[76] Brown SI, Frank TG, Cuschieri A, Sharpe R, Cartwright C. Optimization of the projection screen in a display system for minimal access surgery. Surgical Endoscopy. 2003, 17:1251-1255. 
[77] Brown SI, White C, Wipat K, Hanna GB, Frank TG, Cuschieri A. Characterizing the "Gold Standard" image for laparoscopic surgery. Surgical Endoscopy. 2004, 18:1192-1195.

[78] Szold A. Seeing is believing: visualization systems in endoscopic surgery (video, HDTV, stereoscopy, and beyond). Surgical Endoscopy. 2005, 19(5):730-733.

[79] Van Veelen MA, Meijer DW, Goossens RHM, Snijders CJ, Jakimowicz JJ. Improved usability of a new handle design for laparoscopic dissection forceps. Surgical Endoscopy. 2002, 16:201-207.

[80] Lafreniere R, Berguer R, Seifert P, Belkin M, Roth S, Williams K, Maria E, Napolitano L. Preparation of the operating room. ACS Surgery: Principles and Practice. 2003:1-14.

[81] Sutton E, Youssef Y, Meenaghan N, Godinez C, Xiao Y, Lee T, Dexter D, Park A. Gaze disruptions experienced by the laparoscopic operating surgeon. Surg Endosc. 2010, 24(6):1240-1244.

[82] Franson R, Khabbaz FH, Kapoor A, Patriciu A. A system for laparoscopic surgery ergonomics and skills evaluation. Journal of Endourology. 2011, 25(7):1111-1114. 



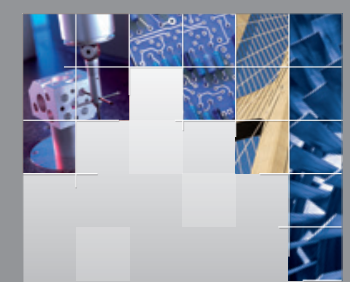

\section{Enfincering}
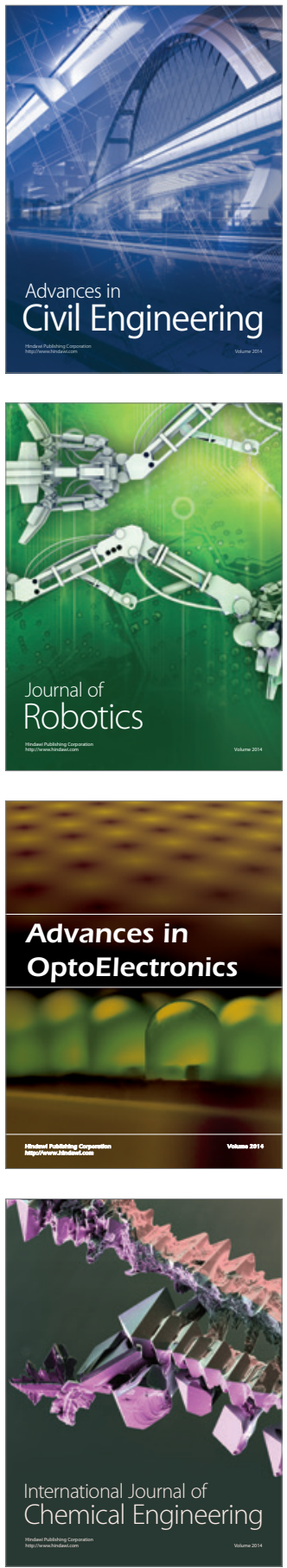

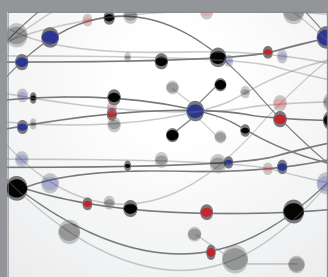

The Scientific World Journal

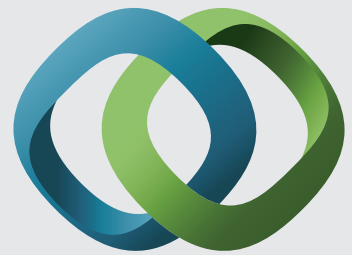

\section{Hindawi}

Submit your manuscripts at

http://www.hindawi.com
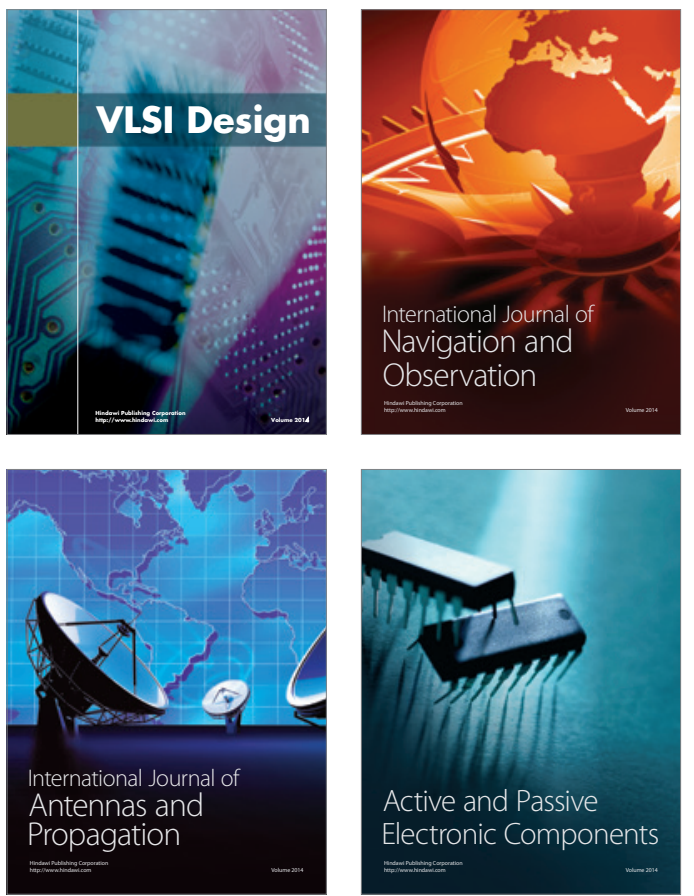
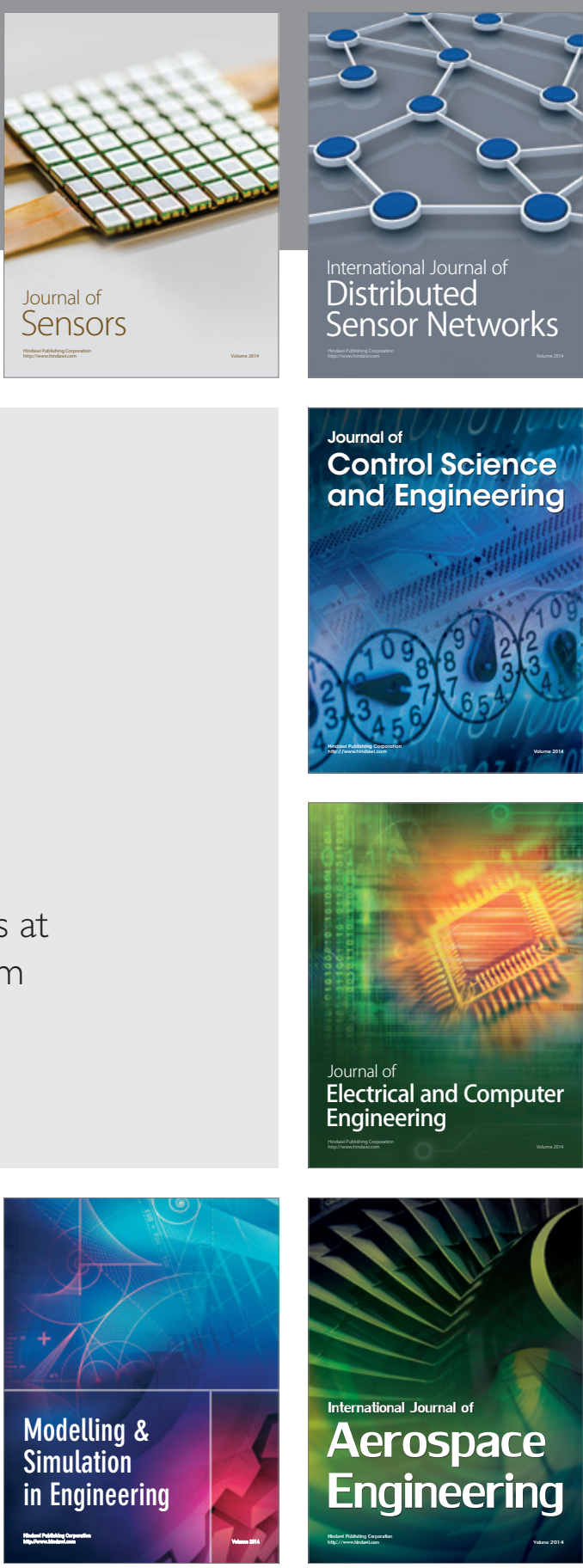

International Journal of

Distributed

Sensor Networks

Journal of

Control Science

and Engineering
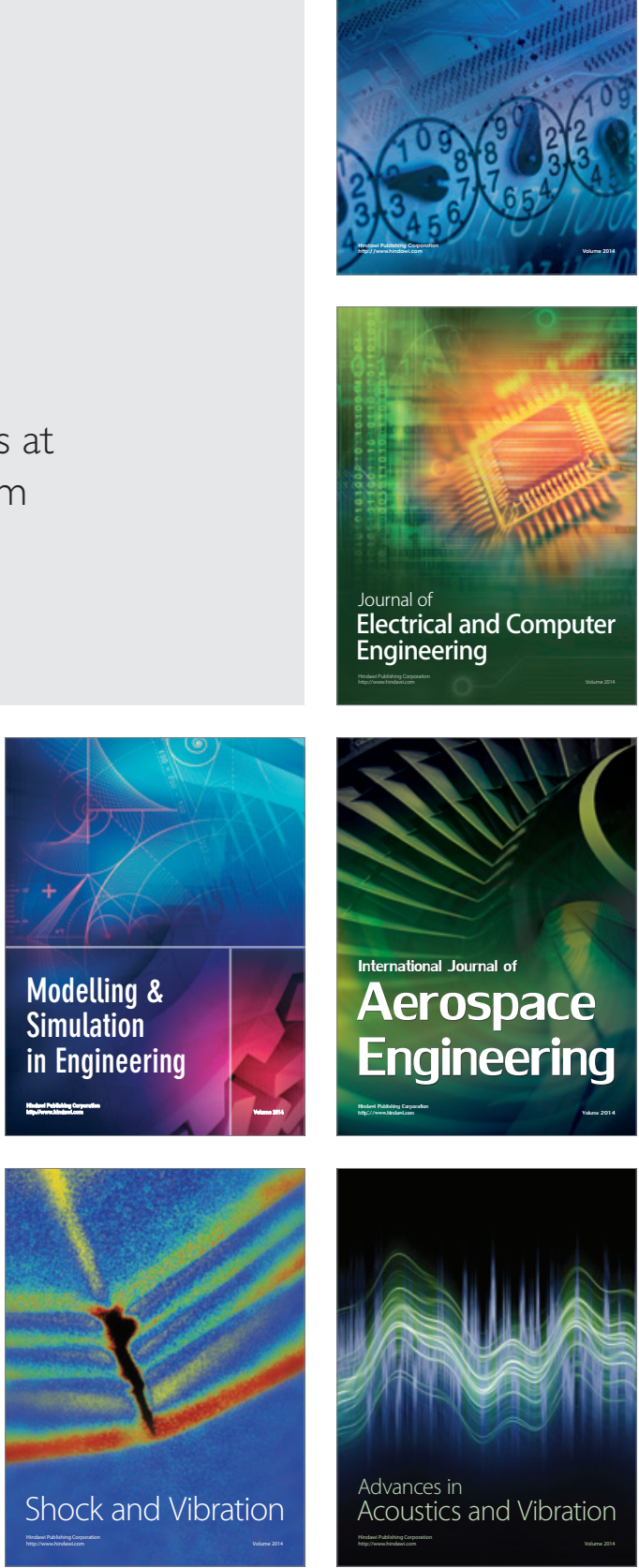